\title{
Using Intentional Contact to Achieve Tasks in Tight Environments
}

\author{
J. Rogelio Guadarrama-Olvera, Emmanuel Dean and Gordon Cheng*
}

\begin{abstract}
Skin technology enabled a powerful way to sense the environment in robotic systems. It allows simplifying the formulation of safety tasks such as collision avoidance between the robot, the environment and surrounding objects. In this paper, a hierarchy policy based on tactile feedback is proposed to let a robot interact with its environment while performing a set of tasks. Such policy lets the safety tasks as collision avoidance and physical interaction, be reduced to simple potential field rules fed directly with tactile feedback which keeps computation demand low. In this context, the concept of "Intentional Contact" is introduced to escape from classic undesired equilibrium points produced by local minima in the potential fields. Allowed contact with the environment empowers a robot to modify its surroundings in order to fulfil the main task. Such contact is permitted as long as the generated force remains under a specific limit, otherwise, a reactive action is taken to reduce it. This new concept is validated in simulation and on a real robot.
\end{abstract}

\section{INTRODUCTION}

In recent years, a number of new robot applications have emerged for tasks where, until now, it was not possible to have a robot. The main reasons are safety [1], reliability [2] or simply because the available technology could not make it feasible. Some of these new applications require tasks where a robot must physically interact with people and dynamically changing environments, for example medical robotics [3], [4], robot-assisted manufacture [5], home assistance robotics [6], [7] and social interaction [8].

Interaction with humans in unstructured environments, require robots to have a reliable way to perceive the surrounding objects to prevent collisions. In addition, a robot must avoid collisions with its own body and most importantly, when human beings are present, it must behave compliantly to external stimuli during the process, [9], [10].

As a strategy to handle these interactions, force-torque control methods have emerged to soften the rigid body structure of a robotic arm [11], [12] and [13]. However, this kind of implementations requires precise modelling, complex control laws, and high-cost sensors and actuators. In this context, hardware-compliant sensors and actuators using spring-based joints and soft materials have been designed to absorb sudden contacts and impacts among the links of a robot as [14], [15]. The concept of robot skin has also been developed to sense contact forces on the links and take actions to induce compliant behaviour on stiff robot architectures. Different sensing technologies have been utilized for skin purposes as in [16], [17], [18] and [19].

\footnotetext{
* All the authors are with the Institute for Cognitive Systems, Electrical Engineering Faculty, Technical University of Munich. rogelio.guadarrama@tum.de, dean@tum.de, gordon@tum.de, www.ics.ei.tum.de
}

For mechanically less complicated robot architectures, the problem of avoiding self-collisions and impacts with the environment has been tackled using model-based approaches as [20], [21], [22] and [23]. These implementations require a geometric mapping of the robot and the environment to evaluate repulsive potential functions between the present bodies. Simplified geometric models are often used in such implementations to reduce the computing demand and also to ensure a non-interaction virtual layer surrounding all the body-parts and detected objects. The success of this approach relies on an accurate perception of the environment, then for unstructured and changing scenarios, complex perception systems are required which increase the computing demands.

Potential fields are widely used in robotics to prevent collisions [21], [24]. However, choosing a proper function to define the vector fields may become difficult. Local minima can get the trajectory stuck into undesired equilibrium points trying to avoid contacts as showed in [25]. Such scenario may keep the robot from accomplishing other tasks, for instance, to track a certain position or orientation on the end effector.

Some strategies to escape from local minima have been proposed as the virtual obstacle approach in [26] or the hierarchical execution of tasks in [27]. Within this hierarchical approach, collision avoidance can be defined as a task between other goal and safety tasks.

There are different methods to achieve the execution of a set of tasks according to established hierarchy. For instance, null space projection methods use the remaining degrees of freedom available after fulfilling all the higher priority tasks to accomplish lower priority tasks. This can be done by defining null-space projectors between the tasks' workspaces as in [28] and [29]. Another way to implement this approach is using a chain of quadratic problems using the results of a higher priority task as restrictions for lower priority tasks [30] and [31].

In this paper, a task hierarchy policy is proposed to manage task priorities according to tactile data provided by multi-modal robot skin. Such policy allows reducing the safety tasks as collision avoidance and physical interference compliance to simple potential field rules. It is well known that potential fields are prone to undesired behaviours such as oscillations and stagnations due to local minima. However, with this scheme, smooth task priority changes are triggered when local minima are detected in presence of obstacles allowing the robot to touch objects on the environment under a defined tolerable force.

The concept of "Intentional Contact" is introduced in this work as a tool to explore and clear mobile obstacles from the environment in order to enable the execution of the goal- 
tasks. Skin force feedback during the Intentional Contact phases lets the policy trigger an emergency rearrangement if the contact force exceeds a permitted rank. In that scenario, reducing the contact force takes the highest priority.

The proposed policy can be applied to any hierarchical task manager on position, velocity or force-controlled robots. The scheme was tested on numerical simulations of a redundant planar manipulator and on our robot TOMM [32] which is a semi-humanoid platform with two 6-DOF arms covered with artificial skin [33].

The paper will follow the next structure: Section II will present the skin-based collision avoidance task based on potential fields. Section III will introduce the concept of "Intentional Contact". Section IV will integrate both tasks on a hierarchical structure according to the proposed priority policy. Section $\mathrm{V}$ is dedicated to testing the proposed policy on a redundant system on numerical simulation. Section VI will present the validation on the real platform and Section VII will present conclusions.

\section{SKIN BASED COLLISION AVOIDANCE}

A standard representation of a robotic system in generalized coordinates is defined as

$$
M(q) \ddot{q}+c(q, \dot{q})=\tau
$$

being $q \in \mathbb{R}^{n}$ the position of the system in generalized coordinates, $\dot{q} \in \mathbb{R}^{n}$ is the velocity and $\ddot{q} \in \mathbb{R}^{n}$ the acceleration also in generalized coordinates. $M(q) \in \mathbb{R}^{n \times n}$ is the inertia matrix, $c(q, \dot{q}) \in \mathbb{R}^{n}$ is a vector composed by all the centrifugal, Coriolis, gravity and friction joint torques and $\tau \in \mathbb{R}^{n}$ is a vector of input torques.

Following a standard potential field obstacle avoidance scheme [20]. Every object on the environment, including all the robot's links, are considered to have a repulsive field normal to the object's surface. Such field used to be described by a vector field defined by the geometry of the object. In this form, when an object approaches a robot's link, a reactive force proportional to the distance between the bodies is applied to keep the robot's body away from it. The reactive force can be transformed into a joint torque vector as

$$
\tau_{d}=J_{d}^{T}(q) F_{d}(d)
$$

where $\tau_{d} \in \mathbb{R}^{n}$ is the induced torque, $F_{d} \in \mathbb{R}^{m}$ is the virtually induced force in task coordinates being $m$ the dimension of the task space, $d \in \mathbb{R}$ is the distance between the surfaces of the link and the object and $J_{d} \in \mathbb{R}^{m \times n}$ is the Jacobian matrix which defines the differential kinematic mapping between the task space and the joint space for the surface point where the sensor is collocated.

All the geometrical calculations regarding the potential fields around the objects can be avoided by directly measuring the distance from a link's surface to any surrounding object as shown in Fig. 1. This measurement is possible thanks to the inclusion of a range finder array as in [34]. In addition, because of nowadays electronics are smaller, advanced skin implementations such as the artificial skin [33] have narrower distances between the sensors. With this

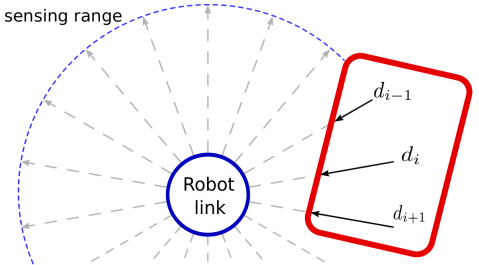

Fig. 1: Direct measurement of repulsive field.

approach, the repulsive force can be calculated defining a sensing range as

$$
F_{d_{i}}=K\left(d_{\text {range }}-d_{i}\right)
$$

where $d_{i}$ is the measured distance by the $i$-th cell of the array and $K \in \mathbb{R}$ a defined repulsion gain. Therefore for a set of $k$ skin cells, the needed torque vector to prevent collisions is defined by

$$
\tau_{d}=\sum_{i=1}^{k} J_{d_{i}}^{T}(q) F_{d_{i}}
$$

This simple potential field rule relays only on the previous knowledge of the position of the skin cells on the surface of the links which is automatically generated using our framework [35]. No previous mapping is required and no knowledge of the surrounding object is required. Additionally, this approach covers both collision avoidance with obstacles on the environment and between the links of the robot.

\section{INTENTIONAL CONTACT}

Let an end effector position controller for (1) be defined as

$$
\tau_{g}=J^{T}(q) F_{g}(x, \dot{x})
$$

where $\tau_{g} \in \mathbb{R}^{n}$ is the torque vector needed to achieve the task, $J \in \mathbb{R}^{m \times n}$ is the Jacobian matrix for the end effector and $F_{d} \in \mathbb{R}^{m}$ is a desired wrench or force in task space produced by a control law to track a desired trajectory of the end effector whose position and velocity are described in task coordinates by $x \in \mathbb{R}^{m}$ and $\dot{x} \in \mathbb{R}^{m}$, respectively. The sub-index $g$ was chosen to denote a goal-task.

On a common implementation, it is expected that the goaltask is executed avoiding any collision. This can be done adding (4) and (5) into the input $\tau$ of (1). However, the usage of added potential fields may lead to local minima producing undesired equilibrium points on the robot trajectory [25]. If the robot's path falls in one of those regions, it would be detected if the norm of the velocity vector $\dot{x}$ decreases to a value lower than a desired transitory threshold $\epsilon_{v}$. In such case, switching of the control law must be considered, for instance, a reduction in the sensing range allowing the robot to approach to objects in order to carry out the goal task.

If the sensing range is decreased to zero and the goal task is still not fulfilled, then the robot can exert forces on the environment to modify its configuration in order to clear the path for the goal task. Following this idea and taking 
advantage of the capabilities of the artificial skin, a robot can exert Intentional Contacts on the surrounding objects and reallocate them to make the goal task feasible. Tactile feedback during the contact makes possible to keep all the contact forces below a defined margin $\epsilon_{c}$ and step back if it is exceeded.

The force limit may be defined by the resilience of the skin layer and also the surrounding objects. Therefore, the system should be able to push and move light objects while fulfilling all the other tasks and reactively respect all the hard physical constraints of the environment. To ensure this, the skin should be able to measure the contact force knowing exactly the point where it is being applied on the robot's body in order to compute a response torque similar as (4)

$$
\tau_{c}=\sum_{i=1}^{k} J_{c_{i}}^{T}(q) F_{c_{i}}
$$

where $\tau_{c} \in \mathbb{R}^{n}$ is the needed torque vector to relax the contact force, $F_{c} \in \mathbb{R}^{m}$ is the contact force measured in task space and $J_{c_{i}} \in \mathbb{R}^{m \times n}$ is the Jacobian matrix which defines the differential kinematic mapping between the task space of dimension $m$ and the joint space of dimension $m$ for the surface point where the force is located at the $i$-th sensed contact point in a set of $k$ measuring points.

\section{HIERARCHICAL TASK ARRANGEMENT}

While executing a set of given tasks, a robot must avoid any contact with the environment. Nevertheless, if this condition is not possible, then Intentional Contact should be allowed as long as the contact force remains below a tolerated level. Furthermore, if the contact force exceeds the allowed value, the control law should take this into account and reduce it. Therefore, the concepts of collision avoidance and contact reaction can be defined as tasks within a defined set of goal-tasks as introduced in [27]. According to the proposed behaviour, three priority arrangements shall be considered as shown in table I.

The conditions in Table I are used for triggering transitions between the three cases. Here, smooth transition methods can help to keep joint torques under feasible values and to avoid overshoot behaviours and chattering on the robot performance which could lead to damage of the mechanical components. Smooth transition techniques as [36] and [37] are useful for this purpose.

\section{EVALUATION BY SIMULATION}

In order to corroborate that the proposed policy matches the desired behaviour, a simulated environment was set with a planar 7-DOF robot. The robot was provided with skin-like sensing capabilities to measure contact forces and proximity on every link, emulating the functionality of artificial skin. Two goal-tasks are defined. The higher priority task is to reach and keep a desired position with the end effector, keeping the velocity bounded during long displacements. This control law is described in [20], where a desired speed
TABLE I: Priority arrangements for obstacle avoidance and Intentional Contact

(a) Transitory phases with collision avoidance

\begin{tabular}{ccl|l}
\hline Priority & Task id & Task name & Conditions \\
\hline 1 & $\tau_{d}$ & Obstacle avoidance & $0 \leq\left\|F_{d}\right\|$ \\
2 & $\tau_{g}$ & Goal task set & $\epsilon_{v}<\|\dot{x}\|$ \\
3 & $\tau_{c}$ & Contact reaction & $\left\|F_{c}\right\|=0$ \\
\hline
\end{tabular}

(b) Intentional approach and contact

\begin{tabular}{ccl|c}
\hline Priority & Task id & Task name & Conditions \\
\hline 1 & $\tau_{g}$ & Goal task set & $0<\left\|F_{d}\right\|$ \\
2 & $\tau_{c}$ & Contact reaction & $\|\dot{x}\| \leq \epsilon_{v}$ \\
3 & $\tau_{d}$ & Obstacle avoidance & $0 \leq\left\|F_{c}\right\|<\epsilon_{c}$ \\
\hline
\end{tabular}

(c) Hard contact reaction

\begin{tabular}{ccl|c}
\hline Priority & Task id & Task name & Conditions \\
\hline 1 & $\tau_{c}$ & Contact reaction & $0 \leq\left\|F_{d}\right\|$ \\
2 & $\tau_{g}$ & Goal task set & $0 \leq\|\dot{x}\|$ \\
3 & $\tau_{d}$ & Obstacle avoidance & $\left\|F_{c}\right\| \geq \epsilon_{c}$ \\
\hline
\end{tabular}

$\dot{x}_{d}$ is defined with respect to the distance to an attracting point in Cartesian space

$$
\dot{x}_{d}=\frac{k_{p}}{k_{v}}\left(x_{d}-x\right)
$$

where the $k_{p}$ and $k_{v}$ are proportional and derivative gains scalars respectively. With this desired end effector speed, a force vector is defined

$$
F_{g}=-k_{v}\left(\dot{x}-\nu \dot{x}_{d}\right)
$$

where the speed saturation function is defined as

$$
\nu=\min \left(1, \frac{V_{m} a x}{\sqrt{\dot{x}_{d}^{T} \dot{x}_{d}}}\right)
$$

being $V_{m} a x$ a maximum speed value allowed for the system.

The second task is to keep the end effector oriented vertically, pointing in the positive direction of $y-$ axis. For this task, the control law is the same but considering the end effector's orientation. A movable obstacle is placed between the initial position of the robot and the desired end effector position to induce a local minimum on the execution of the tasks.

The proposed task-priority policy was implemented using the augmented Jacobian null-space projection described in [29] with soft transitions defined as

$$
\tau=\chi \tau_{k-1}+(1-\chi) \tau_{k}
$$

where

$$
\chi=\frac{\left(1-\tanh \left(\delta_{t}\left(t-t_{t}\right)\right)\right)}{2}
$$

with $\tau_{k-1}$ being the output torque at the moment when the transition was triggered, $\tau_{k}$ the output torque with the new priority arrangement, $t$ the time, $t_{t}$ the specific instant the transition was triggered and $\delta_{t}$ a smoothing gain.

In Fig. 2 is plotted the interaction between the robot and the obstacle while the tasks are executed. The forces 
acting on the robot and the transitions between the task arrangements during the process are shown in Fig. 3.
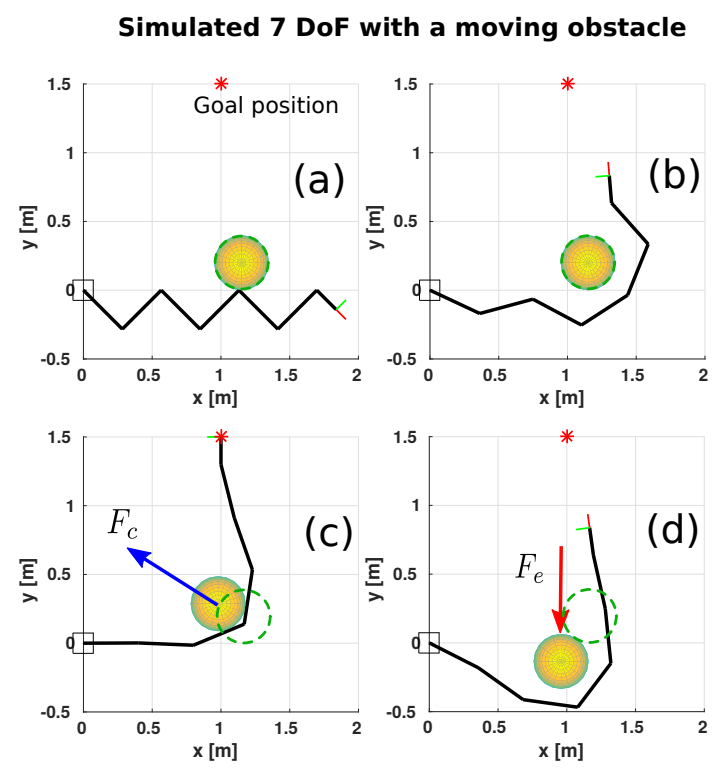

Fig. 2: Stages of the proposed skin reactive policy. (a) Initial condition, the robot's first reaction is to avoid the obstacle. (b) The robot tries to reach its end effector goal position but falls into a local minimum due to the potential fields nature. (c) Intentional contact, the robot approaches and pushes the obstacle with a contact force $F_{c}$ lower than the permissible threshold $\epsilon_{c}$ in order to fulfil the goal-task, the original pose of the obstacle is marked by the dashed circle. (d) Hard contact reaction, an external force $F_{e}>\epsilon_{c}$ is applied to the obstacle so it moves and transfers the force to the robot which moves to reduce the contact force.

\section{EXPERIMENTAL EVALUATION}

In order to evaluate the proposed concept on a physical platform, a robot manipulator covered with skin is needed. It is necessary that the skin is able to measure both contact force and proximity in a reasonable range. Our robot TOMM [32] covers those requirements. It consists of a mobile base with two 6-DOF arms. Both arms are covered with artificial skin provided with sensors to measure acceleration, force, distance, and temperature, on a distributed cellular scheme.

In this experiment, the mobile base was fixed to the ground and a position regulation task was implemented for the end effector as described in Section V. The regulation task will track the desired position with the end effector of TOMM's right arm over a table. With these conditions, two scenarios will be tested. In the first one (Fig. 5), non-fixed obstacles will be placed in the path to the goal position and the robot will have to move them in order to fulfil the goal-task as long as the contact force remains under the tolerated value. One of the obstacles was placed exactly in the desired position. In the second scenario (Fig. 6), a non-fixed obstacle and a fixed obstacle will be placed on the robot's path specially configured to induce the classic local minimum problem
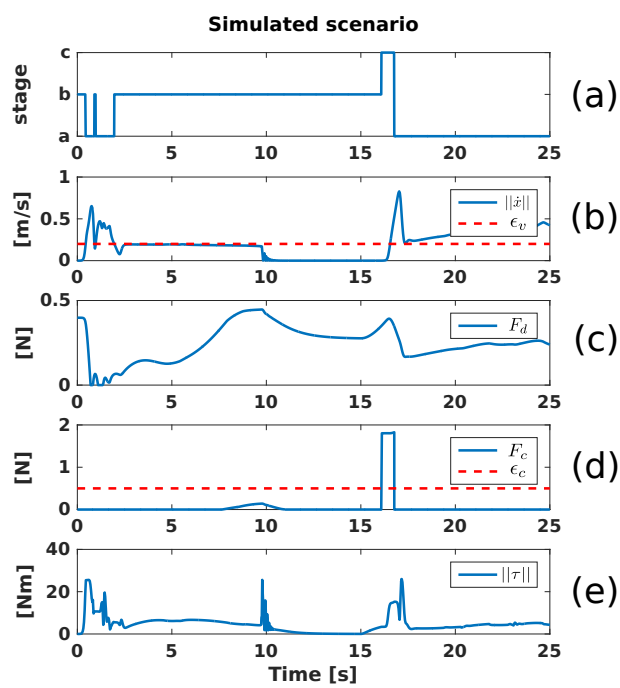

Fig. 3: (a) Priority arrangement according to Table I. (b) End effector speed and minimal value to consider a local minimum in $\left[\frac{\mathrm{m}}{\mathrm{s}}\right]$. (c) Force produced by proximity sensors at the closest point to an obstacle in $[N]$. (d) Highest contact force detected by the skin and maximum permitted contact force in $[N]$. (e) Norm of the torque vector $\tau$ in $[N m]$.

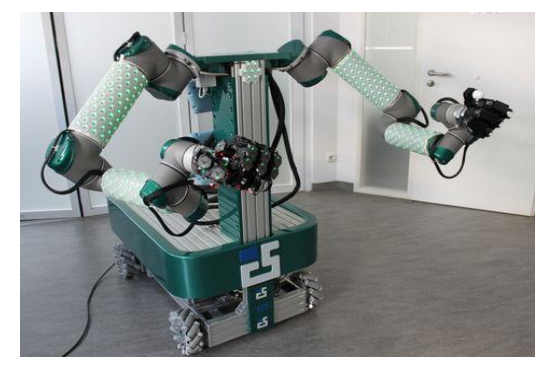

Fig. 4: Experimental platform TOMM, semi-humanoid robot with two 6-DOF arms covered with artificial skin.

using the potential fields. The robot will try to reach the desired position and will face the local minima produced by the obstacles. When the Intentional Contact occurs, the robot will make its path to the desired position by pushing and moving the non-fixed obstacle around the fixed one. The only information provided to the robot in this experiment is the desired position and orientation for the end effector to set the goal task. No information about the obstacles is needed.

In Figures 7 and 8 the interacting forces during the experiment, as well as the priority arrangement configuration according to table I are displayed. In the contact force graph from Fig. 8, a number of Intentional Contact attempts are shown, along the path around the fixed obstacle.

\section{CONCLUSION}

The presented hierarchical policy for interaction provides a reactive way to explore and modify the environment keeping safety as the main concern. It can be implemented under different conditions according to the needs of the 
Experimental setup with non fixed obstacles

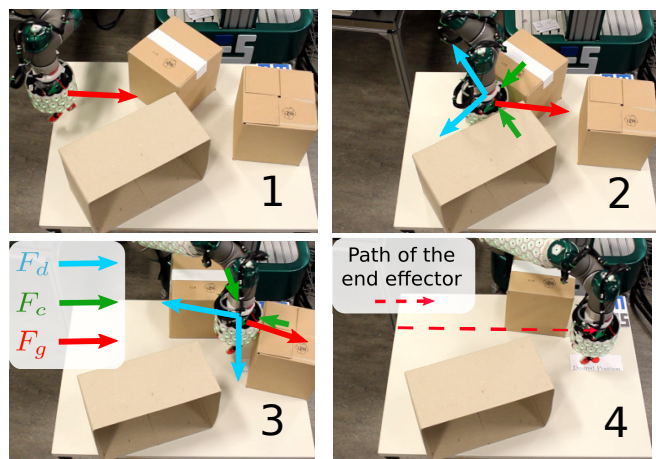

Fig. 5: (1) Initial position. (2) Local minimum reached. (3) Intentional Contact to move the obstacles. (4) Goal position reached. Red arrows describe the Cartesian velocity of the end effector. Blue arrows describe repulsive force generated by the movable objects when they are close to the robot. Green arrows describe de contact forces between the robot and the obstacles. Red arrows describe the attractive force induced by the desired position.

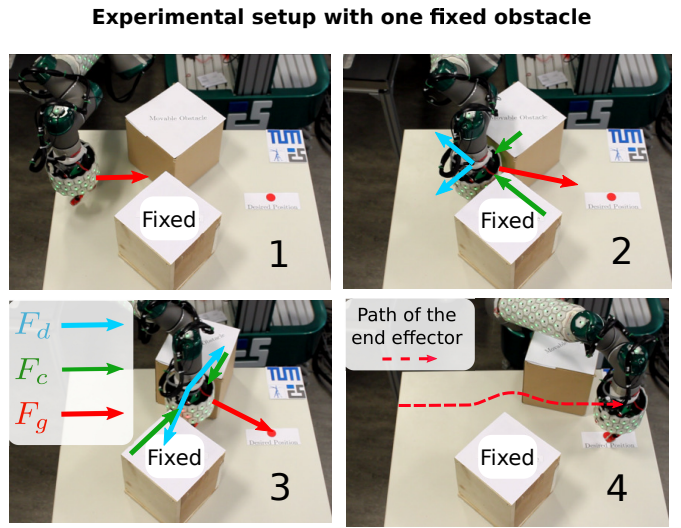

Fig. 6: (1) Initial position. (2) Local minimum reached. (3) Intentional Contact to move the non fixed obstacle. (4) Goal position reached. Red arrows describe the Cartesian velocity of the end effector. Blue arrows describe repulsive force generated by the movable objects when they are close to the robot. Green arrows describe de contact forces between the robot and the obstacles. Red arrows describe the attractive force induced by the desired position.

applications. Other control laws and hierarchy management methods are supported for the proposed set of conditions. The use of potential fields prevents collisions and enables soft interaction contact events to prevent damage to the mechanical parts and the skin itself.

Intentional Contact allows a robot to interact with its environment in order to fulfil the goal tasks. The concept can be applied to other perception technologies but artificial skin expands the idea to the whole body. Tactile feedback plays an important role during Intentional Contact phases because the contact force must be constantly monitored as it is responsible for triggering the hard-contact task priority

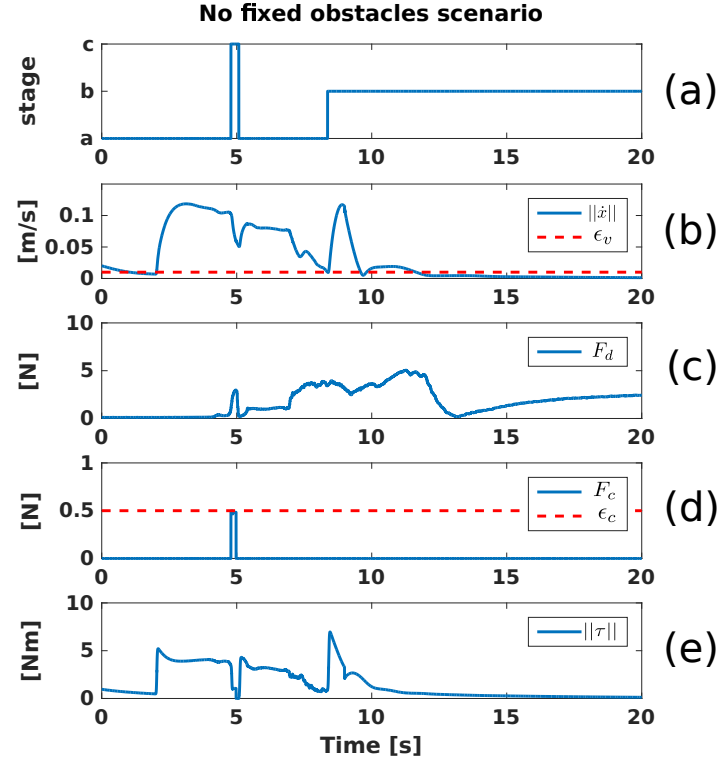

Fig. 7: Movable obstacles scenario. (a) Priority arrangement according to Table I. (b) End effector speed and minimal value to consider a local minimum in $\left[\frac{\mathrm{m}}{\mathrm{s}}\right]$. (c) Force produced by proximity sensors at the closest point to an obstacle in $[N]$. (d) Highest contact force detected by the skin and maximum permitted contact force in $[N]$. (e) Norm of the torque vector $\tau$ in $[\mathrm{Nm}]$.

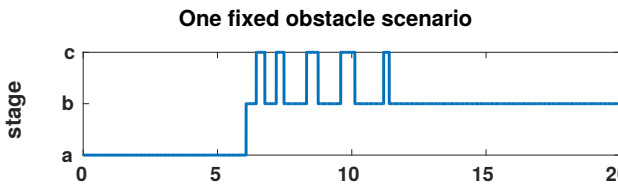

(a)
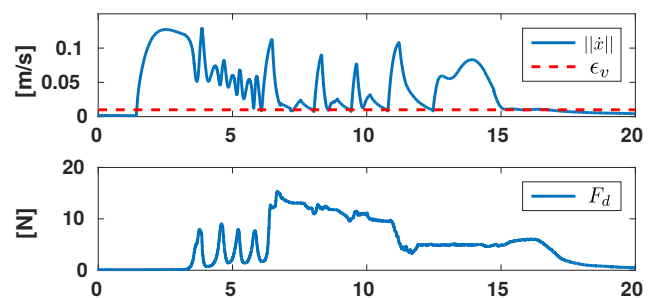

(c)
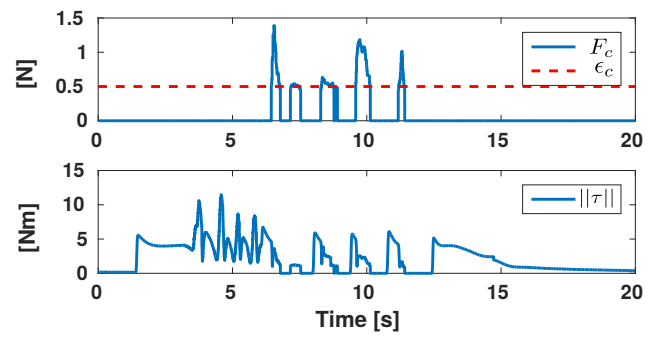

(e)

Fig. 8: Non movable obstacle scenario. (a) Priority arrangement according to Table I. (b) End effector speed and minimal value to consider a local minimum in $\left[\frac{m}{s}\right]$. (c) Force produced by proximity sensors at the closest point to an obstacle in $[N]$. (d) Highest contact force detected by the skin and maximum permitted contact force in $[N]$. (e) Norm of the torque vector $\tau$ in $[\mathrm{Nm}]$. 
arrangement. Enabling Intentional Contact in a robot, make possible to clear the path along unknown scenarios without concerning on the obstacles dynamical properties. The major advantage of this approach is that no previous knowledge about the environment is required. The behaviour of the robot is completely reactive to the environment, task priority rearrangements are triggered by interaction events whose can be also used to modify controller gains and adjust thresholds and tolerances for different operational conditions.

\section{REFERENCES}

[1] S. Haddadin, A. Albu-Schäffer, and G. Hirzinger, "Safety evaluation of physical human-robot interaction via crash-testing." in Robotics: Science and Systems, vol. 3, 2007, pp. 217-224.

[2] O. Agcaoglu, S. Aliyev, H. E. Taskin, S. Chalikonda, M. Walsh, M. M. Costedio, M. Kroh, T. Rogula, B. Chand, E. Gorgun et al., "Malfunction and failure of robotic systems during general surgical procedures," Surgical endoscopy, vol. 26, no. 12, pp. 3580-3583, 2012.

[3] P. Dario, E. Guglielmelli, B. Allotta, and M. C. Carrozza, "Robotics for medical applications," IEEE Robotics \& Automation Magazine, vol. 3, no. 3, pp. 44-56, 1996.

[4] G. Dogangil, B. Davies, and F. R. y Baena, "A review of medical robotics for minimally invasive soft tissue surgery," Proceedings of the Institution of Mechanical Engineers, Part H: Journal of Engineering in Medicine, vol. 224, no. 5, pp. 653-679, 2010.

[5] E. Dean-Leon, K. Ramirez-Amaro, F. Bergner, I. Dianov, P. Lanillos, and G. Cheng, "Robotic technologies for fast deployment of industrial robot systems," in IECON 2016 - 42nd Annual Conference of the IEEE Industrial Electronics Society, Oct 2016, pp. 6900-6907.

[6] B. Graf, M. Hans, and R. D. Schraft, "Care-o-bot iidevelopment of a next generation robotic home assistant," Autonomous robots, vol. 16, no. 2, pp. 193-205, 2004.

[7] K. Yamazaki, R. Ueda, S. Nozawa, M. Kojima, K. Okada, K. Matsumoto, M. Ishikawa, I. Shimoyama, and M. Inaba, "Home-assistant robot for an aging society," Proceedings of the IEEE, vol. 100, no. 8, pp. 2429-2441, 2012.

[8] S. Satake, T. Kanda, D. F. Glas, M. Imai, H. Ishiguro, and N. Hagita, "How to approach humans?-strategies for social robots to initiate interaction," in Human-Robot Interaction (HRI), 2009 4th ACM/IEEE International Conference on. IEEE, 2009, pp. 109-116.

[9] A. Steinfeld, T. Fong, D. Kaber, M. Lewis, J. Scholtz, A. Schultz, and M. Goodrich, "Common metrics for human-robot interaction," in Proceedings of the 1st ACM SIGCHI/SIGART conference on Humanrobot interaction. ACM, 2006, pp. 33-40.

[10] S.-H. Hyon, J. G. Hale, and G. Cheng, "Full-body compliant humanhumanoid interaction: balancing in the presence of unknown external forces," IEEE Transactions on Robotics, vol. 23, no. 5, pp. 884-898, 2007.

[11] M. T. Mason, "Compliance and force control for computer controlled manipulators," IEEE Transactions on Systems, Man, and Cybernetics, vol. 11, no. 6, pp. 418-432, 1981.

[12] A. Albu-Schaffer, O. Eiberger, M. Grebenstein, S. Haddadin, C. Ott, T. Wimbock, S. Wolf, and G. Hirzinger, "Soft robotics," IEEE Robotics \& Automation Magazine, vol. 15, no. 3, pp. 20-30, 2008.

[13] D. E. Whitney, "Historical perspective and state of the art in robot force control," The International Journal of Robotics Research, vol. 6, no. 1, pp. 3-14, 1987.

[14] H. Yu, S. Huang, N. V. Thakor, G. Chen, S.-L. Toh, M. S. Cruz, Y. Ghorbel, and C. Zhu, "A novel compact compliant actuator design for rehabilitation robots," in Rehabilitation Robotics (ICORR), 2013 IEEE International Conference on. IEEE, 2013, pp. 1-6.

[15] B. Vanderborght, N. G. Tsagarakis, R. Van Ham, I. Thorson, and D. G. Caldwell, "Maccepa 2.0: compliant actuator used for energy efficient hopping robot chobino1d," Autonomous Robots, vol. 31, no. 1, pp. 55-65, 2011.

[16] V. J. Lumelsky and E. Cheung, "Real-time collision avoidance in teleoperated whole-sensitive robot arm manipulators," IEEE Transactions on Systems, Man, and Cybernetics, vol. 23, no. 1, pp. 194-203, 1993.

[17] T. Wosch and W. Feiten, "Reactive motion control for human-robot tactile interaction," in Robotics and Automation, 2002. Proceedings. ICRA'O2. IEEE International Conference on, vol. 4. IEEE, 2002, pp. $3807-3812$.
[18] O. Kerpa, K. Weiss, and H. Worn, "Development of a flexible tactile sensor system for a humanoid robot," in Intelligent Robots and Systems, 2003.(IROS 2003). Proceedings. 2003 IEEE/RSJ International Conference on, vol. 1. IEEE, 2003, pp. 1-6.

[19] T. Hoshi and H. Shinoda, "Robot skin based on touch-area-sensitive tactile element," in Proceedings 2006 IEEE International Conference on Robotics and Automation, 2006. ICRA 2006. IEEE, 2006, pp. $3463-3468$

[20] O. Khatib, "Real-time obstacle avoidance for manipulators and mobile robots," The international journal of robotics research, vol. 5, no. 1, pp. 90-98, 1986.

[21] P. Khosla and R. Volpe, "Superquadric artificial potentials for obstacle avoidance and approach," in Robotics and Automation, 1988. Proceedings., 1988 IEEE International Conference on. IEEE, 1988, pp. 1778-1784.

[22] A. De Santis, A. Albu-Schaffer, C. Ott, B. Siciliano, and G. Hirzinger, "The skeleton algorithm for self-collision avoidance of a humanoid manipulator," in 2007 IEEE/ASME international conference on advanced intelligent mechatronics. IEEE, 2007, pp. 1-6.

[23] F. Seto, K. Kosuge, and Y. Hirata, "Self-collision avoidance motion control for human robot cooperation system using robe," in 2005 IEEE/RSJ International Conference on Intelligent Robots and Systems. IEEE, 2005, pp. 3143-3148.

[24] P. Vadakkepat, K. C. Tan, and W. Ming-Liang, "Evolutionary artificial potential fields and their application in real time robot path planning," in Evolutionary Computation, 2000. Proceedings of the 2000 Congress on, vol. 1. IEEE, 2000, pp. 256-263.

[25] Y. Koren and J. Borenstein, "Potential field methods and their inherent limitations for mobile robot navigation," in Robotics and Automation, 1991. Proceedings., 1991 IEEE International Conference on. IEEE, 1991, pp. 1398-1404.

[26] M. G. Park and M. C. Lee, "A new technique to escape local minimum in artificial potential field based path planning," KSME international journal, vol. 17, no. 12, pp. 1876-1885, 2003.

[27] A. Dietrich, T. Wimbock, A. Albu-Schaffer, and G. Hirzinger, "Integration of reactive, torque-based self-collision avoidance into a task hierarchy," IEEE Transactions on Robotics, vol. 28, no. 6, pp. 12781293,2012

[28] N. Mansard, O. Stasse, P. Evrard, and A. Kheddar, "A versatile generalized inverted kinematics implementation for collaborative working humanoid robots: The stack of tasks," in Advanced Robotics, 2009. ICAR 2009. International Conference on. IEEE, 2009, pp. 1-6.

[29] A. Dietrich, C. Ott, and A. Albu-Schäffer, "An overview of null space projections for redundant, torque-controlled robots," The International Journal of Robotics Research, p. 0278364914566516, 2015.

[30] O. Kanoun, F. Lamiraux, and P.-B. Wieber, "Kinematic control of redundant manipulators: Generalizing the task-priority framework to inequality task," IEEE Transactions on Robotics, vol. 27, no. 4, pp. 785-792, 2011.

[31] A. Escande, N. Mansard, and P.-B. Wieber, "Hierarchical quadratic programming: Fast online humanoid-robot motion generation," The International Journal of Robotics Research, p. 0278364914521306, 2014.

[32] E. Dean-Leon, B. Pierce, P. Mittendorfer, F. Bergner, K. RamirezAmaro, W. Burger, and G. Cheng, "TOMM: Tactile Omnidirectional Mobile Manipulator," in 2017 IEEE International Conference on Robotics and Automation (ICRA), Accepted, May 2017.

[33] P. Mittendorfer and G. Cheng, "From a multi-modal intelligent cell to a self-organizing robotic skin," in Proceedings of the IEEE International Conference on Robotics and Automation (ICRA), 2013.

[34] C. Boddy and J. Taylor, "Whole-arm reactive collision avoidance control of kinematically redundant manipulators," in Robotics and Automation, 1993. Proceedings., 1993 IEEE International Conference on. IEEE, 1993, pp. 382-387.

[35] E. Dean-Leon, F. Bergner, K. Ramirez-Amaro, and G. Cheng, "From multi-modal tactile signals to a compliant control," in 2016 IEEE-RAS 16th International Conference on Humanoid Robots (Humanoids), Nov 2016, pp. 892-898.

[36] N. Mansard, A. Remazeilles, and F. Chaumette, "Continuity of varying-feature-set control laws," IEEE Transactions on Automatic Control, vol. 54, no. 11, pp. 2493-2505, 2009.

[37] M. Liu, Y. Tan, and V. Padois, "Generalized hierarchical control," Autonomous Robots, vol. 40, no. 1, pp. 17-31, 2016. 